\title{
Development of a non-contact torque transducer based on the laser speckle contrast method
}

\author{
Alberto Garinei ${ }^{1}$ and Roberto Marsili $^{2}$ \\ ${ }^{1}$ DMII Department, Università degli Studi Guglielmo Marconi, 00193 Rome, Italy \\ ${ }^{2}$ Dipartimento di Ingegneria, Università degli Studi di Perugia, Via Duranti, no. 1, 06125 Perugia, Italy \\ Correspondence to: Roberto Marsili (roberto.marsili@unipg.it)
}

Received: 20 May 2017 - Revised: 21 June 2017 - Accepted: 22 June 2017 - Published: 14 July 2017

\begin{abstract}
When a torque measurement is required, torque transducers show many drawbacks during their use: the usual limits are the need for contact and the effects on shaft line parameters. A new approach is proposed in this work: a non-contact torque meter for a machine shaft has been developed. It carries out torque measurements evaluating the torsional displacement between two distinct sections of the shaft, through the monitoring of their roughness.

The sensing principle employed is the classical laser speckle contrast method. The outcoming intensity scattered by the rough surface is processed by a cross-correlation function. In this way, the angular torsion of two distinct sections is obtained. It is therefore possible to point out the applied torque knowing the shaft torsional stiffness.
\end{abstract}

\section{Introduction}

In order to carry out the torque measurement $M$, an indirect measurement technique has been applied. Considering two distinct sections of a shaft, and knowing the torsional stiffness module $K$, the value of the torque applied is obtained by the following equation, where $\Delta \vartheta$ is the torsion of two sections of the shaft:

$M=K \cdot \Delta \vartheta$

The innovating technique consists of a new optical experimental evaluation of the angular shift, different from the traditional technologies, even the ones employing optical instrumentation, such as stereoscopic lights. The technique is substantially based on the laser speckle contrast method, commonly employed in roughness measurement, as shown in Toh et al. (1998, 2001), Wang et al. (1998), Leonard and Toal (1998) and Yarlagadda (2010). In our application it is used in a torque evaluation supported by the cross-correlation technique for time shift.

Considering two rotating points in two different shaft sections, the roughness profiles will obviously show a periodic trend. In an unloaded configuration, a cross-correlation (Yarlagadda, 2010) of the two roughness profiles will carry out a function with a maximum in time equal to $t_{\mathrm{dw}}$. If a torque is applied, the two sections will undergo a reciprocal rotation. Therefore the previous roughness profiles will be translated in the time domain. Performing again a crosscorrelation, it will get out a time function, characterized by a maximum after a period equal to $t_{\text {up }}$. These crosscorrelation maxima can be used to extrapolate the angular rotation through the following relation, which also needs the rotational velocity $\omega$ monitoring, in the unloaded and uploaded configurations.

$$
\Delta \vartheta=\left(\frac{t_{\mathrm{up}}}{\omega_{\mathrm{up}}}\right)-\left(\frac{t_{\mathrm{dw}}}{\omega_{\mathrm{dw}}}\right)
$$

Combining Eqs. (1) and (2), the applied torque is obtained (Speranzini et al., 2016).

Recent works which brought to the interferometric techniques, such Chang et al. (1998) and Kandpal et al. (2000), have provided input for the development of the proposed method; this represents a new approach for torque measurements, because it allows no-contact torque measures, which do not interfere with the machine rotor dynamics as in the classical approaches for torque measurements that involve the use of strain gauges (Myers et al., 2013), accelerometers (D’Emilia et al., 2015a, 2016), surface acoustic wave (SAW) 


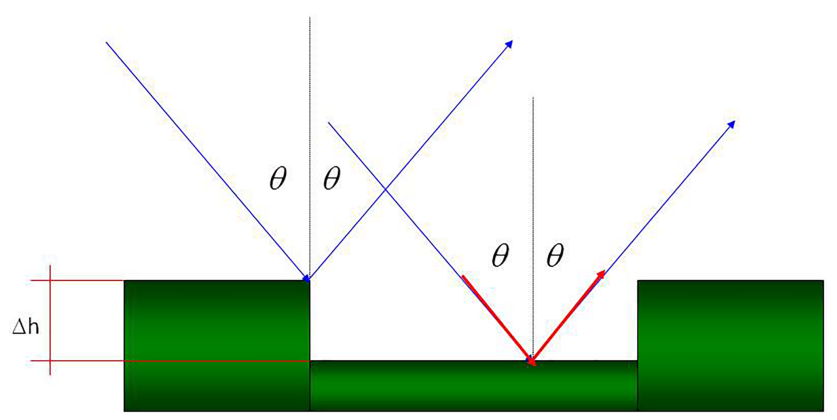

Figure 1. Rayleigh roughness definition.

sensors, or a magnetic-encoder-ring or Hall effect sensing element (Cardelli et al., 2015; Fleming, 2008), that may also have problems of thermal stability (Partin et al., 2006; D'Emilia et al., 2015b). It is important to underline how such a measurement is completely contact-free, and it provides the signal only thanks to an incident coherent light on the shaft, coming out from optical fiber. The proposed technique is easier to be applied than other non-contact measurement techniques used for the characterization of materials and mechanical components, such as image correlation (Garinei and Marsili, 2013; Speranzini and Agnetti, 2014) and thermo-elasticity (Becchetti et al., 2009a, b). Those techniques permit the performance of "full field" stress and strain measurements, but they need high-performance dataprocessing and image-acquisition systems (Brustenga et al., 2005) and elaborate post-processing procedures to perform analysis on rotating mechanical components (Becchetti et al., 2010; Garinei and Marsili, 2014; Speranzini and Agnetti, 2013).

The outline of this paper is as follows. First, the laser speckle contrast method is described in detail, and the performances of the laser contrast method and cross-correlation repeatability tests are discussed in different conditions simulated on a specific test bed. Then the realized measurement setup for torque measurements is described and tested.

\section{The scattering of a coherent light by a rough surface: the speckle}

As reported in Beckmann and Spizzichino (1987), a possible distinction between rough and smooth surfaces can be made by focusing the attention on their properties of light scattering. In Fig. 1, a light beam, characterized by a wavelength $\lambda$ and incoming on a step surface, is considered.

Let us consider the reflected light from two different planes, distant $\Delta h$. The optical path of the two beams is different, as is evident in Fig. 1. This difference is geometrically obtained and equal to the following:

$$
\Delta r=\frac{2 \Delta h}{\cos \theta}
$$

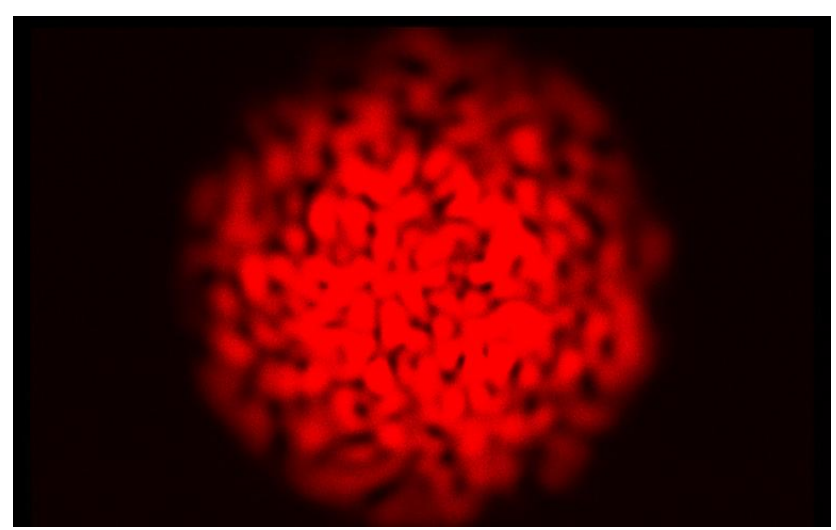

Figure 2. A typical distribution of speckle.

The step represented in the figure could be considered as a simplified roughness profile, whose relationship with the phase shift could be determined by the following proportion:

$2 \pi: \lambda=\Delta \phi: \Delta r$.

In Eqs. (3) and (4), two extreme cases occur: when the waves are in phase and when the waves are in phase opposition. In the first condition, the light would be reflected as by a smooth surface, while in the second case the two rays of Fig. 1 would combine themselves destructively. Just to respect the energy conservation principle, the incident light should therefore be scattered in all the other directions, as it occurs with rough surface. Conventionally the limit value is fixed at $\pi / 2$, just to distinguish between roughness and smoothness, for which the following condition, for the light wavelength, is derived:

$\Delta h<\frac{\lambda}{8 \sin \theta}$.

Equation (5) defines the detectable roughness with a light probe of wavelength $\lambda$.

Employing an appropriate source, it is possible to produce an interaction between rough surface and scattered light-ray phases. One of the possible ways to work out this effect is the well-known laser speckle contrast technique. Indeed, macroscopically, the different phase shift produced by the incidence of a light spot on a rough surface results in a diffuse reflection composed of light and dark points, forming the so-called speckle; see Fig. 2. The speckle distribution varies with roughness as a function of phase shift. It is then possible to get speckle's related information, and consequently roughness to, channelling the scattered light into an optical fiber. By coupling the fiber with a photodiode, an electric signal is obtained.

With this setup for the roughness monitoring of a rotating surface, a typical resulting roughness profile in the time domain is shown in Fig. 3, in which the presence of the periodicity peaks is evident. 


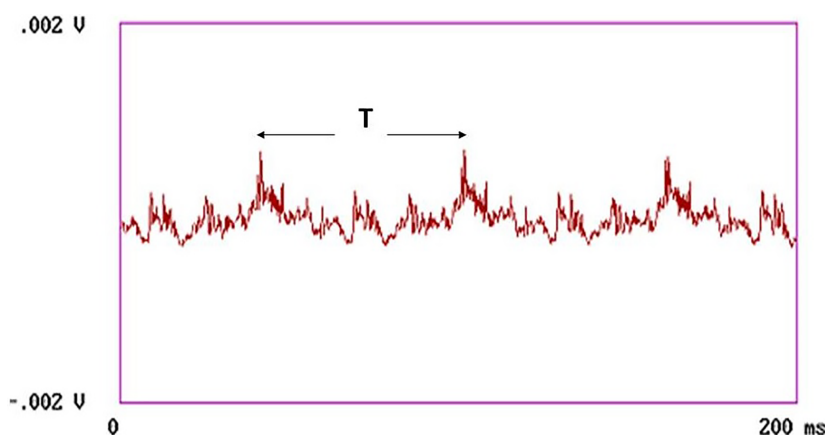

Figure 3. Typical roughness-dependent intensity function.

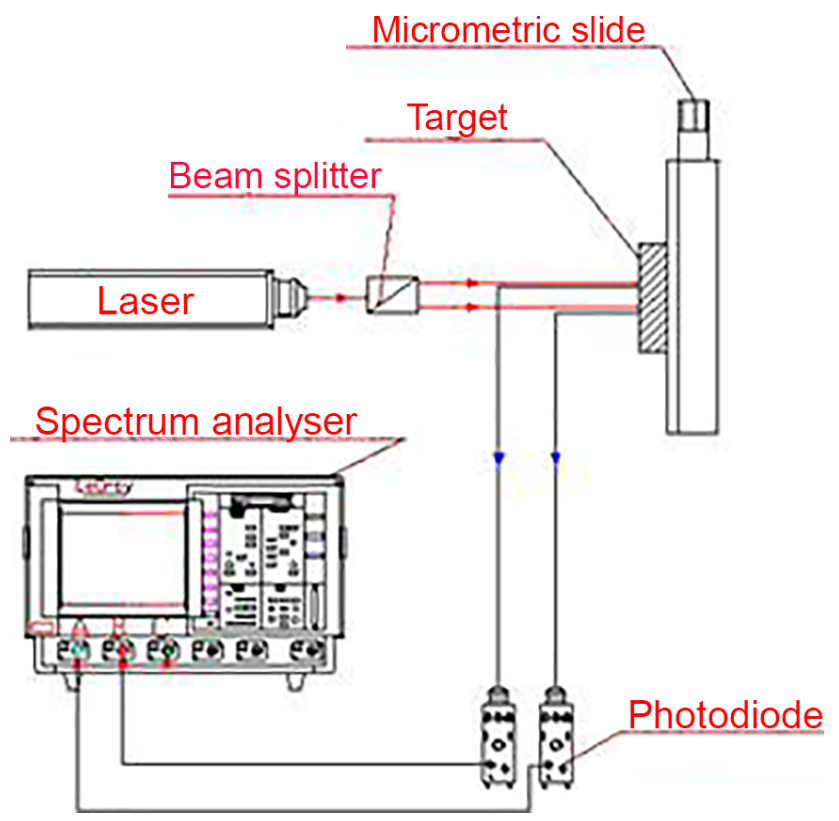

Figure 4. Sketch of the measurement chain.

\section{Study of the performances of the laser contrast method measurement chain}

The realized measurement chain is illustrated in Fig. 4. The first step was the optimization of the experimental setup. A $10 \mathrm{~mW} \mathrm{He}-\mathrm{Ne}$ laser at $632 \mathrm{~nm}$ wavelength as a light source and four multi-mode optical fibres have been used. The outcoming light from the laser is split, through an in-fibre beam splitter, in two multi-mode fibres, whose light strikes the target surface. The target is made of a steel plate with a known roughness, measured by a traditional mechanical instrument. The target is mounted on an electromechanical sledge, which permits both horizontal and vertical translation with $1 \mu \mathrm{m}$ steps. The back-reflected light is received by other two multimode fibres, which bring the light up to two photodiodes and then to a spectrum analyzer.

The two sensing heads are basically fiber optic reflection sensors, focalized by a graded index cylindrical lens. There-

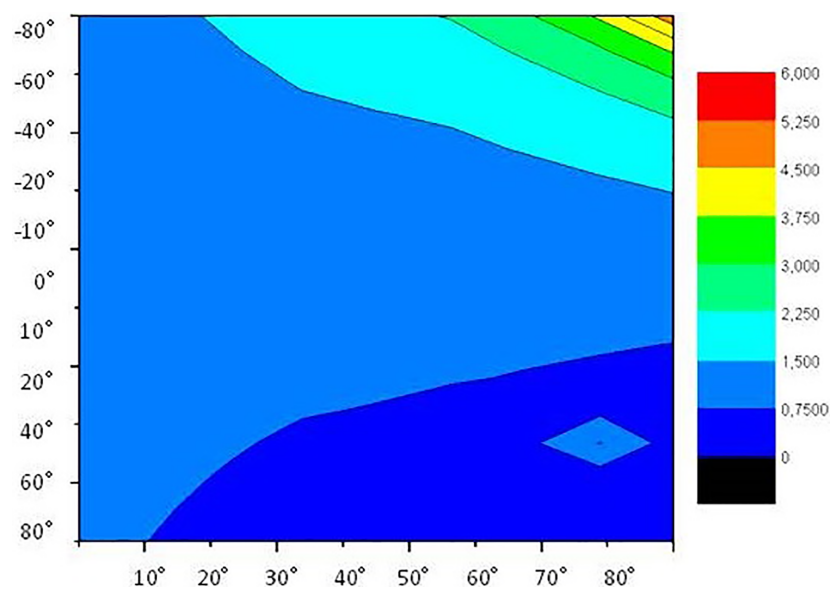

Figure 5. Scattering coefficient dependence on the incident and reflection angle of the light.

fore it was necessary to calibrate these sensing heads to verify the misalignment's effect.

The theoretical analysis highlights that better test conditions can be realized by increasing the incident angle value $\theta$ up to $90^{\circ}$, as shown in Fig. 5. This means that the optical fibre couplers should be placed as near as possible to the grazing angle with respect to the target surface. In this way, the signal intensity would be optimized.

However, the worst conditions have been tested, to check the instrument performance: the fibres have been positioned at nearly $\theta \approx 10^{\circ}$.

The first optical fiber is mounted on a mechanical micrometric sledge while the second is fixed. In this way it is also possible to realize the spatial resolution evaluation of the described setup. In fact, by moving the sledge, an increment of the distance between the fibre couplers is produced. By checking the voltage profile changes, or the cross-correlation peaks, as mentioned above, the spatial resolution is obtained.

The incoming light beams have been focused through "selfoc" lenses and spacers and then experimentally detected by moving the horizontally target sledge until the detection of the backscattered signal's intensity maximum.

The curve shown in Fig. 6 presents a maximum around $19 \mathrm{~mm}$. The performances of the measurement chain have been studied under this configuration.

The first tested feature is the repeatability. The target has been moved along the vertical direction at $3 \mathrm{~mm} \mathrm{~s}^{-1}$ and a cross-correlation of the signals coming from photodiodes have been performed. The results are shown in Fig. 7 .

In four different tests, the cross-correlation function follows a common profile, with an increasing inaccuracy around the zero value of the function. The divergence maximum is at $500 \mathrm{~ms}$ with a standard deviation equal to $15 \%$ of the reading value. This is highly reduced around the peak where the standard deviation is $2 \%$ of the reading value. This highlights the 


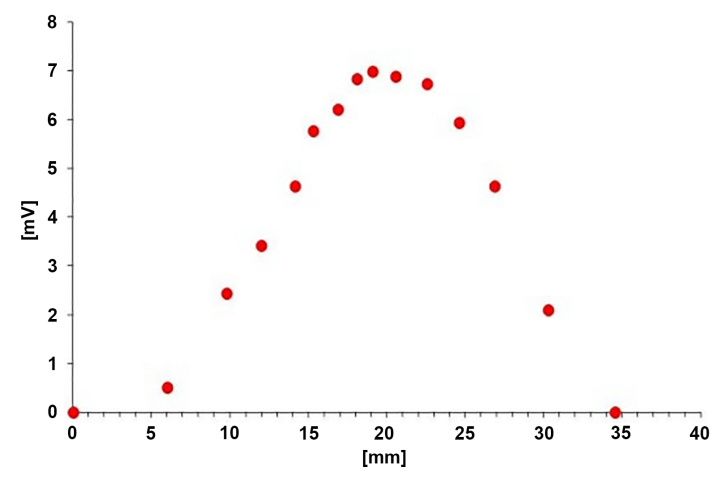

Figure 6. Back-reflected light intensity against fibre head or target distance.

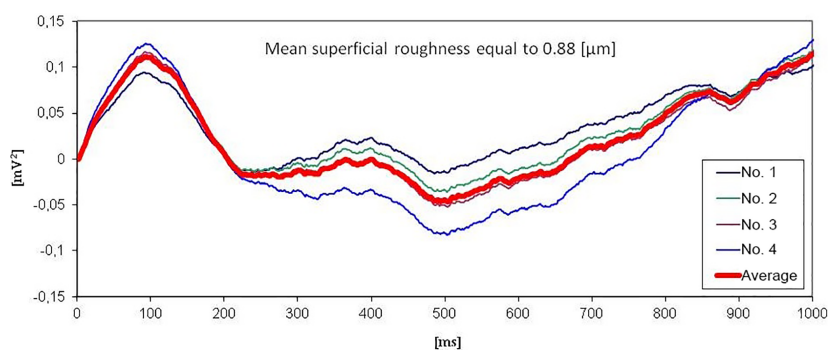

Figure 7. Cross-correlation repeatability test.

need to follow the temporal shift of the first cross-correlation peak in order to measure in the best test conditions.

In a second test, the distance between the fibre couplers have been increased by $10 \mu \mathrm{m}$ for the spatial-resolution evaluation of the instrument. This second test, realized in such conditions, highlights that the system is sensitive to the distance between the fibres: indeed the cross-correlation function changes, as shown in Fig. 8.

However, successive tests pointed out the spatial resolution dependence from the following factors: target roughness, incident angle value, incident light wavelength and material type.

As mentioned in the theoretical analysis, the first three factors modulate the speckle distribution and intensity along the observation direction, while the material knowledge is critical due to the dependence of the scattered light from the conductibility (Myers et al., 2013).

As result of the performed tests, the system spatial resolution results equal to $10 \mathrm{~mm}$. However, the possibility of enhancing such a feature is quite reasonable, just by optimizing the measurement conditions. In particular, in order to improve the signal-to-noise ratio some improvement can be made: the environmental light interference should be reduced as well as the misalignments which cause the light losses, the angle of the fibre couplers should be optimized, and the lightsource power should be increased, so that a higher spatial resolution could be obtained. However, the spatial resolution obtained is sufficient to carry out the prototype testing.

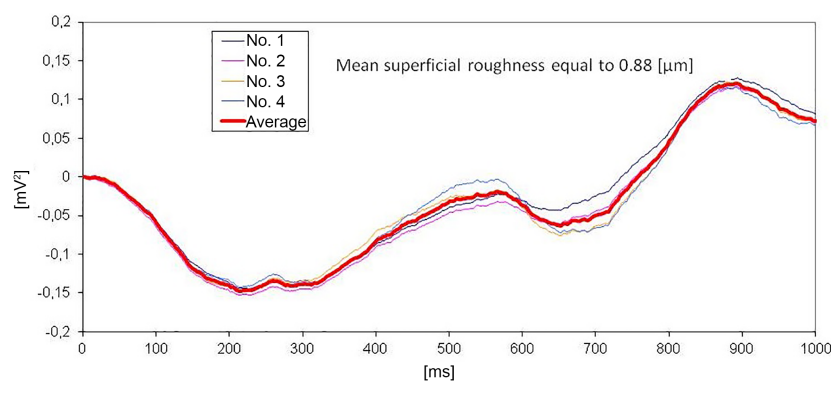

Figure 8. Cross-correlation $(\mu \mathrm{s})$.

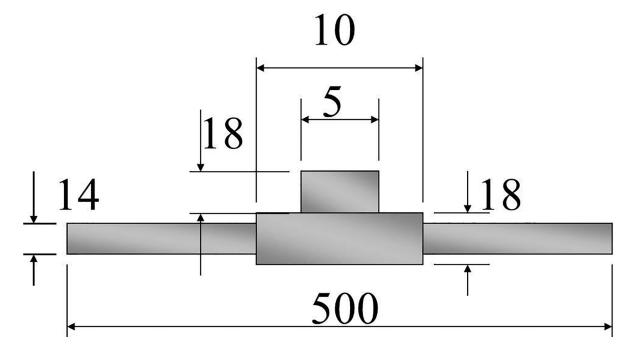

Figure 9. The shaft (dimensions in millimetres, $\mathrm{mm}$ ).

\section{The measurement setup and the experimental results}

Once the measurement chain features are defined, it is possible to create an ad hoc setup in order to monitor the noncontact torque measurement technique feasibility. The simplest shaft configuration has been used and reported in Fig. 9; it is characterized by a mean superficial roughness equal to $0.88 \mathrm{~mm}$, the same as that tested before. The material is aluminium.

This is mounted between an electric three-phase asynchronous $0.75 \mathrm{~kW}$ motor and a linear electromagnetic brake. This assembly is represented in Fig. 10.

The applied torque is constantly monitored by a piezoelectric load cell.

Under this configuration, the measurement of the applied torque has been performed through Eqs. (1) and (2).

In order to know the shaft stiffness to deduce the torque from the angular torsion, a finite element method (FEM) shaft model has been created (Fig. 11).

In this case, the shaft simplicity only permits the obtainment of the theoretically necessary information. However, in order to define a general procedure for any kind of shaft, the numerical way has been preferred. The stiffness value is deduced by the model applying a known torque and recording the angular torsion of the shaft. Giving as input the same values tested in the experimental phase, a comparison between the numerical and experimental results is possible. The torsional stiffness is then deduced. 


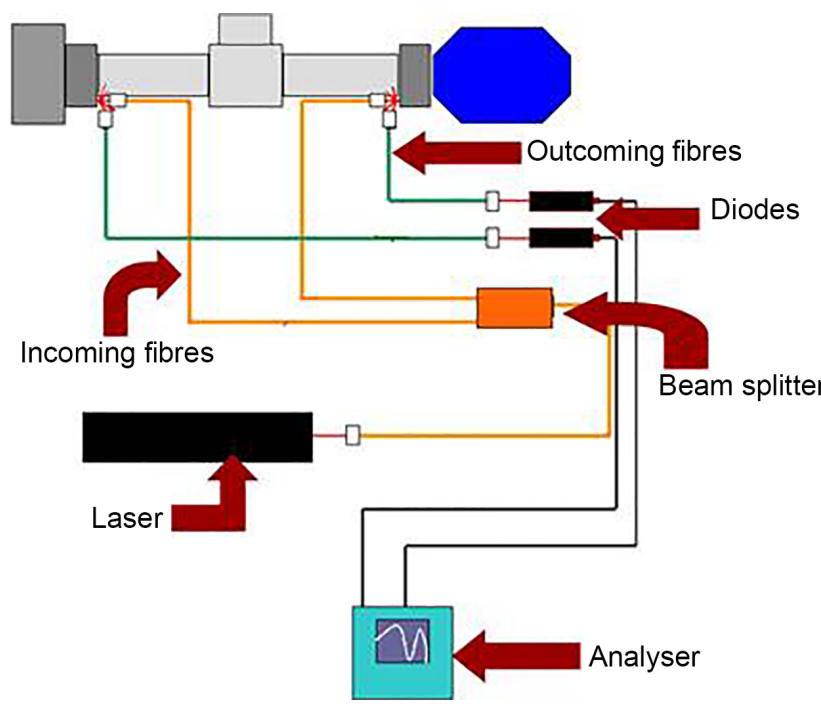

Figure 10. The measurement setup.

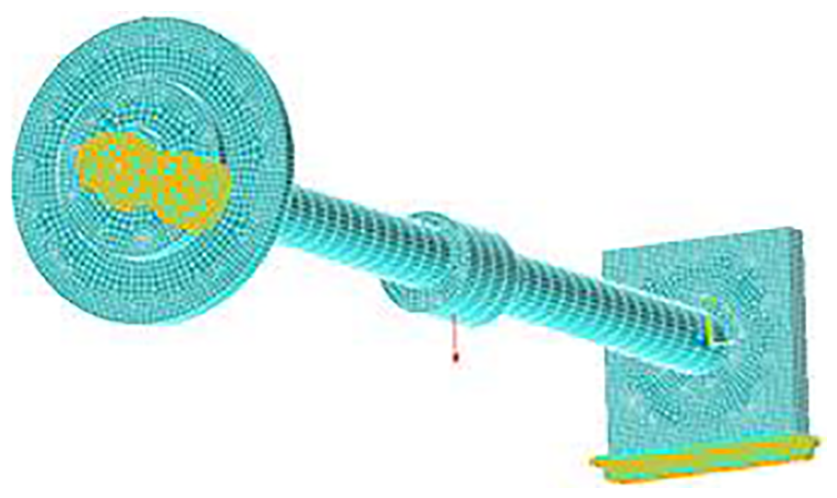

Figure 11. The shaft model.

The cross-correlation and the auto-correlation functions, shift for different applied torques, and the angular velocity and the angular shift are reported in Table 1.

Using the torsional stiffness value in Eq. (1), the torques measured by the optical chain are reported in Tables 2 and 3 , in comparison with the corresponding numerical outputs.

Analysing the results shown in the previous tables, it is apparent that there is a fundamental aspect to emphasize: if the numerical angular shift is greater than the experimental one, the applied torque measurement is less than numerical torque value, and vice versa. It appears to be, therefore, a correlation between these two data sets. The standard deviation value $\sigma$ of the points of calibration has resulted (for a range of torque between 1 and $10 \mathrm{~N} \mathrm{~m}$ ) that is equal to $1.3 \mathrm{~N}$. The uncertainty relative to the full scale was lower than $13 \%$.
Table 1. Experimental results.

\begin{tabular}{lrrr}
\hline Cradle torque measurement & $1(\mathrm{~N} \mathrm{~m})$ & $3(\mathrm{~N} \mathrm{~m})$ & $8(\mathrm{~N} \mathrm{~m})$ \\
\hline Cross-correlation $\Delta T(\mathrm{~ms})$ & $3.72 \times 10^{-2}$ & 0.265 & 0.53 \\
Auto correlation $\Delta T(\mathrm{~ms})$ & 60.5 & 61 & 64.63 \\
Angular velocity $\left(\mathrm{rad} \mathrm{s}^{-1}\right)$ & 103.8 & 102.8 & 97 \\
Angular shift $(\mathrm{rad})$ & $3.8 \times 10^{-3}$ & 0.0275 & 0.051 \\
\hline
\end{tabular}

Table 2. Torque measurement results.

\begin{tabular}{lcc}
\hline $\begin{array}{l}\text { Test } \\
\text { number }\end{array}$ & $\begin{array}{c}\text { Input } \\
\text { torque } \\
\text { value } \\
\left(\mathrm{N} \mathrm{m}^{-1}\right)\end{array}$ & $\begin{array}{c}\text { Experimental } \\
\text { torque } \\
\text { measurement } \\
\left(\mathrm{N} \mathrm{m}^{-1}\right)\end{array}$ \\
\hline 1 & 1.0 & 0.6 \\
2 & 3.0 & 4.2 \\
3 & 8.0 & 7.6 \\
\hline
\end{tabular}

Table 3. Angular shift comparison.

\begin{tabular}{lrr}
\hline $\begin{array}{l}\text { Applied } \\
\text { torque } \\
\left(\mathrm{N} \mathrm{m}^{-1}\right)\end{array}$ & $\begin{array}{r}\text { Numeric } \\
\text { angular } \\
\text { shift } \\
(\mathrm{rad})\end{array}$ & $\begin{array}{r}\text { Experimental } \\
\text { angular shift } \\
(\mathrm{rad})\end{array}$ \\
\hline 1 & $6.6 \times 10^{-3}$ & $3.8 \times 10^{-3}$ \\
3 & 0.0199 & 0.0275 \\
8 & 0.052 & 0.051 \\
\hline
\end{tabular}

\section{Conclusions}

As has been pointed out in the feature evaluation phase, the optical instrument proposed is able to detect the angular shift through the monitoring of the superficial roughness in two different sections of the shaft. The auto- and cross-correlation function in a downloaded state is used to obtain the torque from the torsional shift and from the shaft stiffness. This latter feature is obtainable both theoretically, for simple configurations, and numerically through a FEM program. Moreover, the comparison between the numerical expected values and the experimental results is a powerful tool for the model validation and, consequently, for the evaluation of different materials and shapes. The instrument innovation is given by the possibility to perform a non-contact torque measurement. At the same time, the measurement chain dimensions could be significantly reduced by using miniaturized optical components, or, at least, the optical fibres bringing the roughness measurement away enough to not interfere with the test environment.

Even if these tests have been realized with an ad hoc setup, the results give the chance to develop similar systems whenever a torque measurement is required. In this sense, many further developments are possible. The power of the light 
source and its wavelength, the relative position of the emitting and receiving fiber and the optical alignment improvement using fiber coupled components are all fundamental aspects which are able to significantly enhance the system performances in a wide field of application.

Data availability. No data sets were used in this article.

Competing interests. The authors declare that they have no conflict of interest.

Edited by: Rosario Morello

Reviewed by: two anonymous referees

\section{References}

Becchetti, M., Flori, R., Marsili, R., and Rossi, G. L.: Stress and strain measurements by image correlation and Thermoelasticity, in: SEM Annual conference \& Exposition on Experimental and Applied Mechanics, 1-4 June 2009, Albuquerque, New Mexico, USA, 2009a.

Becchetti, M., Flori, R., Marsili, R., and Rossi, G. L.: Measurement of stress and strain by a thermocamera", in: SEM Annual conference \& Exposition on Experimental and Applied Mechanics, 1-4 June 2009, Albuquerque, New Mexico, USA, 2009b.

Becchetti, M., Flori, R., Marsili, R., and Moretti, M.: Comparison between Digital Image Correlation and Thermoelasticity for Strain Field Analysis, in: 9th International conference on Vibration measurements by laser and noncontact techniques, 2225 June 2010, Ancona, https://doi.org/10.1063/1.3455462, 2010.

Beckmann, P. and Spizzichino, A.: The scattering of Electromagnetic Waves from Rough Surface, Artech House, Incorporated, Artech House, Norwood, Massachusetts, 1987.

Brustenga, G., Marsili, R., Moretti, M., Pirisinu, J., and Rossi, G.: Measurement on rotating mechanical component by thermoelasticity, J. Appl. Mech. Mater., 3-4, 337-342, https://doi.org/10.4028/www.scientific.net/AMM.3-4.337, 2005.

Cardelli, E., Faba, A., Marsili, R., Rossi, G., and Tomassini, R.: Magnetic nondestructive testing of rotor blade tips, J. Appl. Phys., 117, 17A705, https://doi.org/10.1063/1.4907180, 2015.

Chang, D., Merat, F. L., Discezo, F. M., and Harris, J. S.: Neural net based torque sensor using birefrangent material, Sensor Actuat. A, 70, 243-249, 1998.

D’Emilia, G., Di Rosso, G., Gaspari, A., and Massimo, A.: Metrological interpretation of a six sigma action for improving on line optical measurement of turbocharger dimensions in the automotive industry, Proceedings of the Institution of Mechanical Engineering: Part D, J. Auto. Eng., 229, 261-269, https://doi.org/10.1177/0954407014539671, 2015a.

D'Emilia, G., Gaspari, A., and Natale, E.: Dynamic Calibration Uncertainty of Three-Axis Low Frequency Accelerometers: Test Rig and Procedure Aspects, Acta Imeko, 4, 75-81, 2015b.
D’Emilia, G., Gaspari, A., and Natale, E.: Evaluation of aspects affecting measurement of three-axis accelerometer, Measurement, 77, 95-104, 2016.

Fleming, W. J.: New Automotive Sensors, IEEE Sensors J., 8, 1900-1921, https://doi.org/10.1109/JSEN.2008.2006452, 2008.

Garinei, A. and Marsili, R.: Design of an optical measurement system for dynamic testing of electrospindles, Measurement, 46, 1715-1721, https://doi.org/10.1016/j.measurement.2013.01.006, 2013.

Garinei, A. and Marsili, R.: Thermoelastic Stress Analysis of the Contact Between a Flat Plate and a Cylinder, Measurement, 52, 102-110, https://doi.org/10.1016/j.measurement.2014.03.005, 2014.

Kandpal, H. C., Mehta, D. S., and Vaishya, J. S.: Simple method for measurement of surface roughness using spectral interferometry, Opt. Lasers Eng., 34, 139-148, 2000.

Leonard, L. C. and Toal, V.: Roughness measurement of metallic surfaces based on the laser speckle contrast method, Opt. Lasers Eng., 30, 433-440, 1998.

Myers, D. R., Chan, M. W., Vigevani, G., Sosnowchik, B. D., Azevedo, R. G., Jog, A. V., Lin, L., and Pisano, A. P.: Torque measurements of an automotive halfshaft using microdoubleended tuning fork strain gauges, Sensors Actuat. A, 204, 79-87, 2013.

Partin, D. L., Heremans, J. P., Schroeder, T., Thrush, C. M., and Flores-Mena, L. A.: Temperature Stable Hall Effect Sensors, IEEE Sensors J., 6, 106-110, https://doi.org/10.1109/JSEN.2005.860362, 2006.

Speranzini, E. and Agnetti, S.: Post-cracking behaviour of reinforced glass beams, in: Proceedings of COST Action TU0905, Mid-term conference on structural glass, 18-19 April 2013, Poreč, Croatia, 2013.

Speranzini, E. and Agnetti, S.: The technique of digital image correlation (DIC) to identify defects in glass structures, Struct. Cont. Health Monit., 21, 2015-2029, https://doi.org/10.1002/stc.1629, 2014.

Speranzini, E., Agnetti, S., and Corradi, M.: Experimental analysis of adhesion phenomena in fibre-reinforced glass structures, Composites Pt. B, 101, 155-166, https://doi.org/10.1016/j.compositesb.2016.06.074, 2016.

Toh, S. L., Shang, H. M., and Tay, C. J.: Surface Roughness Study Using Laser Speckle Method, Opt. Lasers Eng., 29, 217-225, 1998.

Toh, S. L., Quan, C., Woo, K. C., Tay, C. J., and Shang, H. M.: Whole field surface roughness measurement by laser speckle correlation technique, Opt. Laser Technol., 33 427-434, 2001.

Wang, W., Wong, P. L., Luo, J. B., and Zhang, Z.: A new optical technique for roughness measurement on moving surface, Tribol. Int., 31, 281-287, 1998.

Yarlagadda, R. K. R.: Analog and Digital Signals and Systems, Springer, Boston, MA, USA, 2010. 